\title{
A Single Electron Transistor Made From Lead Cadmium Selenide Nanocrystals
}

\author{
Parikshit Sahatiya \\ Department of Physics and Nanotechnology, SRM University, SRM Nagar, Kattankulathur -603203
}

\begin{abstract}
With the miniaturization of transistor size, fabrication of transistor with lithography technique is a foreseen problem. It is possible to routinely create semiconductor nanocrystals whose dimensions are much smaller than those can be realized by lithography techniques using colloidal chemistry. The size of the nanocrystals can be varied easily by varying the process parameters to study the quantum confinement effect. Here we present the simple synthesis method of Pb-CdSe and characterization of the same with XRD, UV-VIS spectroscopy and AFM. Also we present the I-V characteristics of Single Electron Transistor, by applying different values of Gate voltage. Decrease in the size of the nanoparticles will result in increase in charging energy and hence leads to more prominent Coulomb Blockade phenomena. Gate voltage controls the movement of electron from the blockade region and hence can be used in charge sensing application as they detect the motion of single electron.
\end{abstract}

Keywords: Single electron transistor, Lead Cadmium Selenide, Coulomb Blockade, IV characteristics, AFM

\section{INTRODUCTION}

With miniaturization of transistor size, following Moore law is becoming a bit difficult. Many sophisticated fabrication techniques are required for fabrication. Single Electron Transistor (SET) is the possible replacement to CMOS and also continuing the Moore law [1]. SET promises small transistor size, low power dissipation, simple fabrication which are currently the major problems in CMOS technology[2][3]. SET is very charge sensitive device, capable of detecting charges far less than one electron. This remarkable property makes SET a very useful tool in experiments where high charge sensitivity is required. They are increasingly used and proposed as measurement device for quantum computation, logic elements and Quantum dot cellular automata [3].

SET consists of two tunnel junctions and a small conducting island which is coupled to source and drains lead by tunnel junctions and capacitively coupled to gate as shown in Fig. 1. The reason for not coupling gate by tunnel junctions is that we need to control the electron in the island by means of gate voltage. Hence capacitance of the gate-island region is made high so that it does not form a tunnel junction

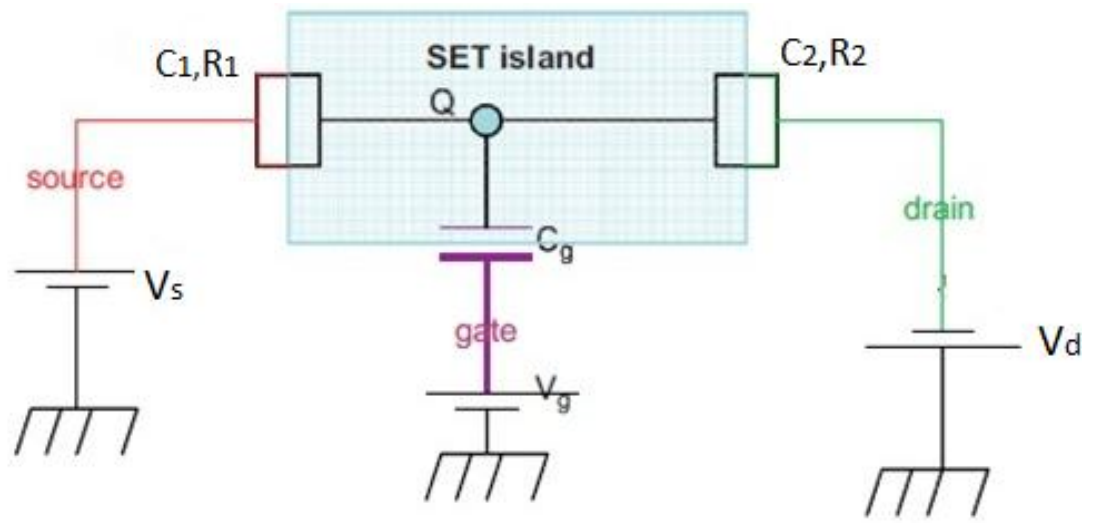

Fig. 1 shows basic electrical representation of SET where tunnel junctions are represented by capacitor connected parallel to resistor 


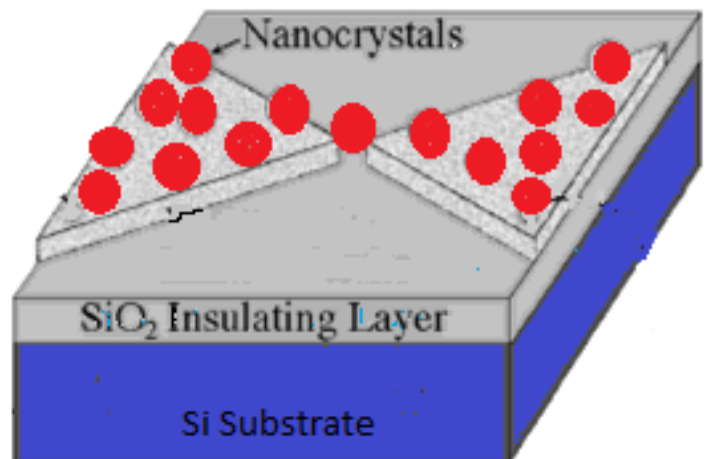

Fig. 2 shows idealized diagram of SET with lead cadmium selenide nanocrytals with size 2.1 nm are spaced to closely bound Au leads

\section{SET PHYSICS}

SET works on the principle of tunneling of charges from source to drain with voltage applied at gate terminal. It exhibits an important phenomena called Coulomb Blockade in which charge carrier will pass the island only after sufficient voltage exist across the capacitor. Tunnel junctions can be modeled as parallel combination of capacitor and tunneling resistance (In Fig. 1, $\mathrm{C}_{1}$ and $\mathrm{R}_{1}$ are the tunneling capacitance and tunneling resistance, similarly for $\mathrm{C}_{2}$ and $\left.\mathrm{R}_{2}\right)$. The effect of this tunneling resistance $\left(\mathrm{R}_{\mathrm{T}}\right)$ should be weak to prevent charge of tunneling electrons to be delocalized over capacitor plates. For this, $\mathrm{R}_{\mathrm{T}}$ should be very large but finite. An approximate analysis of $\mathrm{R}_{\mathrm{T}}$ can be done by uncertainty relation between time and energy which gives the value of $R_{T}>>h / e 2$ to be $25.6 \mathrm{k} \Omega$ [4]. Energy stored in capacitor is given by $E=Q^{2} / 2 C$. For tunneling to occur, energy should be large and capacitance should be less. For macroscopic capacitor $\left(\mathrm{C}=1.1 * 10^{-13} \mathrm{~F}\right)$ the change in energy due to transfer of single electron is $1.16 \mu \mathrm{eV}$ (which is negligible). Nanoscale capacitor $\left(\mathrm{C}=1.1 * 10^{-19} \mathrm{~F}\right)$ yields a change of energy $(1.1 \mathrm{eV})$ which is appreciable fraction of electron volt. The capacitance measured with Lead Cadmium Selenide nanocrystals are in range of $10^{-19}$ F.Hence, these nanocrystals are suitable for the tunneling phenomena.

The charging energy for capacitor is $\mathrm{E}=\frac{\mathrm{Q} 2}{2 \mathrm{C}}$. Now, let a single electron tunnel through the insulating layer from one end to another, energy stored in the field capacitor is $E=(Q+e) 2 / 2 C$.

Change in energy is $\Delta \mathrm{E}=\frac{-\mathrm{e}\left(\mathrm{Q}+\frac{\mathrm{e}}{2}\right)}{\mathrm{C}}$

For tunneling to occur $\Delta \mathrm{E}>0$

Therefore,

$\left(\mathrm{Q}+\frac{\mathrm{e}}{2}\right)>0, \mathrm{Q}>-\mathrm{e} / 2, \mathrm{~V}>-\mathrm{e} / 2 \mathrm{C}$

Energy is conserved and since energy stored decreases upon tunneling the electron ends above Fermi level on the other side of junction. Note that upon tunneling the electron ends above Fermi level on the other side of junction. Note that upon tunneling voltage over the junction will decrease by $|\mathrm{e} / \mathrm{c}|$.

In order for tunneling to occur the condition is,

$-\mathrm{e} / 2 \mathrm{C}<V<e / 2$

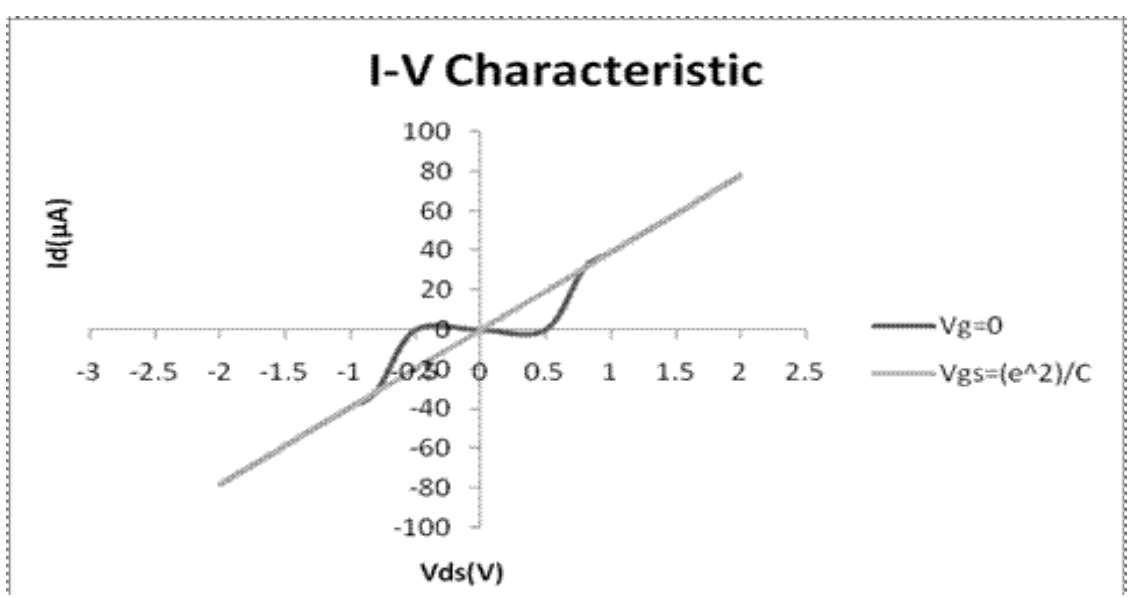

Fig. 3 shows the I-V characteristic of SET with various value of $V_{d s}$. (Resistance of the tunneling junction considered here is minimum which is $25.6 \mathrm{~K} \Omega$ ). When we apply charging energy to gate, $I_{d}$ flows instantaneously 
on application of $V_{d s}$, while if gate voltage is kept zero then it takes energy from $V_{d s}$ to charge the island. The voltage needed to charge the island when $V_{g}=0$ is calculated as $0.72 \mathrm{~V}$

Temperature plays a vital role in coulomb blockade phenomena. Charging energy should be greater than thermal energy to observe coulomb blockade phenomena.

$\Delta \mathrm{E}>\frac{\mathrm{KBT}}{2}$

If we assume " $n$ " number of electrons tunneling then,

$-\frac{\text { ne }}{2 \mathrm{C}}<V<\frac{\mathrm{ne}}{2 \mathrm{C}}$

Therefore, $\mathrm{n}=\sqrt{\frac{\mathrm{CKT}}{\mathrm{e}^{\wedge} \mathrm{T}}}$, where $\mathrm{n}$ is the number of electrons that could be transferred due to thermal energy.

\section{SYNTHESIS OF LEAD CADMIUM SELENIDE}

$0.33 \mathrm{~g}$ of Cadmium acetate and $0.05 \mathrm{~g}$ of Lead acetate were dissolved in $40 \mathrm{ml}$ of ethylene glycol and the solution was heated in an oven for few minutes (3-5 min).Selenium powder $(0.05 \mathrm{~g})$ was added in the solution. The solution was stirred with constant heating at $300^{\circ} \mathrm{C}$ using a magnetic stirrer at the speed of $600 \mathrm{rpm}$. The constant heating and stirring was carried out till a change of color from the initial - dark black to reddish brown was observed. Approximately, the time taken for the color change to appear was around 50 minutes. Once the reddish brown color was obtained the heating and stirring was halted immediately and the solution was allowed to cool down. The product obtained was centrifuged at $4000 \mathrm{rpm}$ for 15 minutes. The resulting product was then dried overnight at $78^{\circ} \mathrm{C}$ using oven.

\section{RESULT\& DiscuSSION}

$X R D$

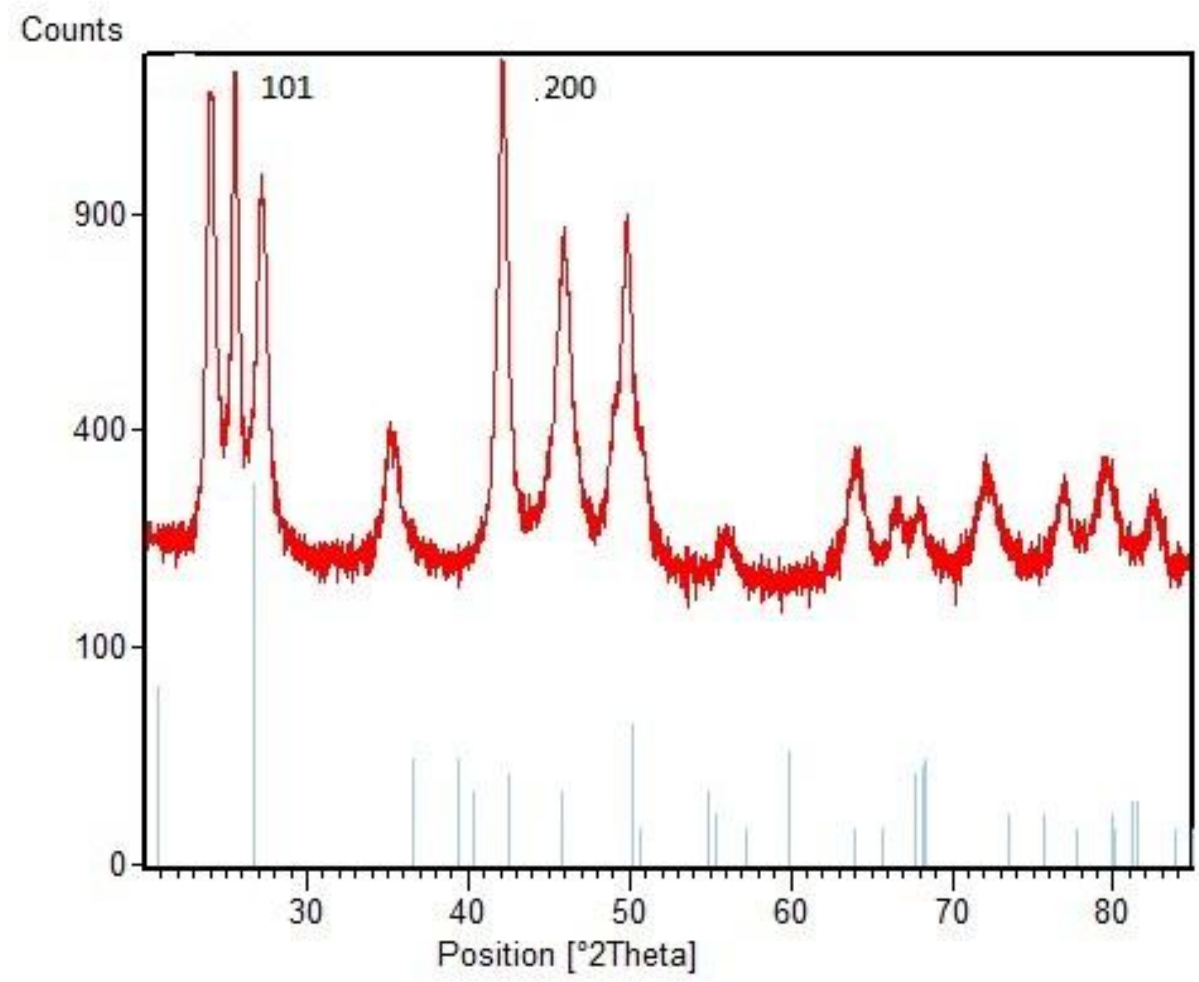

Fig. 4 shows the XRD result of Pb-CdSe which is having peaks at 2 Theta angles of 26.52 and 42.465

\section{UV-VIS}




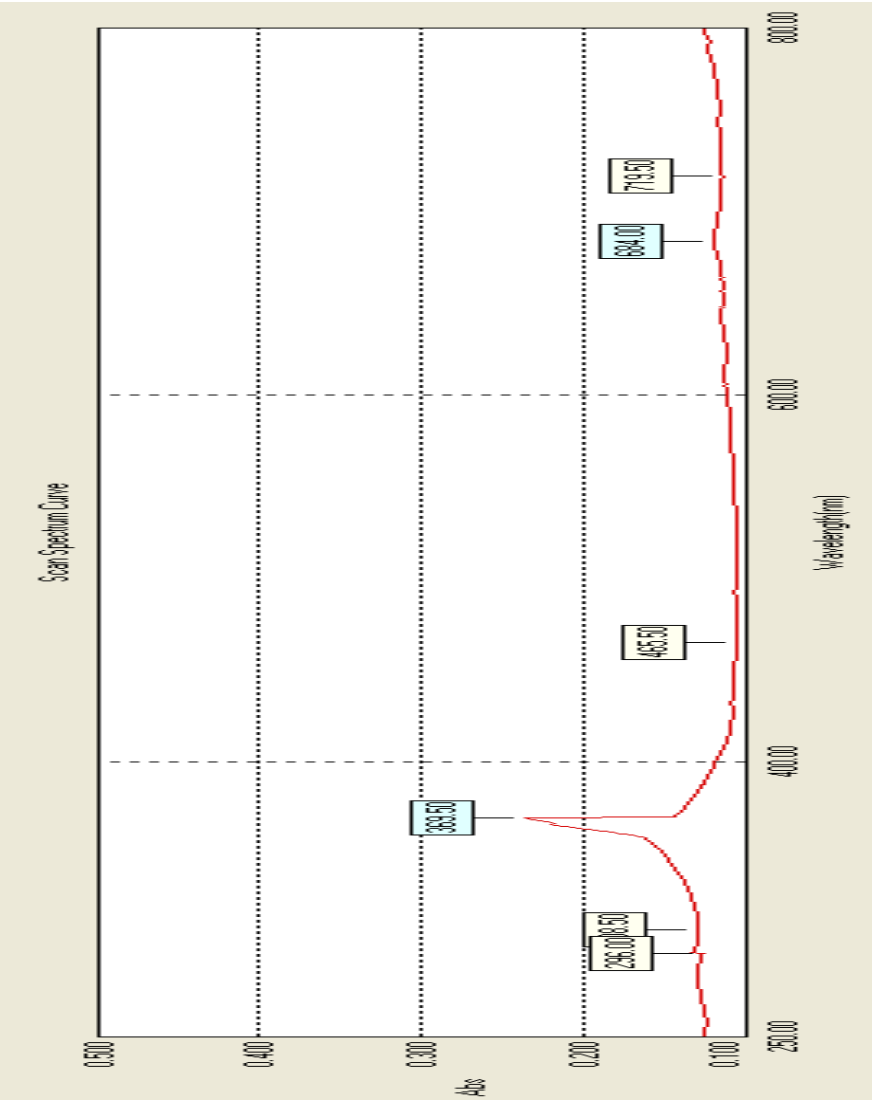

Fig. 5 shows the UV-VIS spectroscopy result of Pb-CdSe nanoparticles which has absorbance at $684 \mathrm{~nm}$.
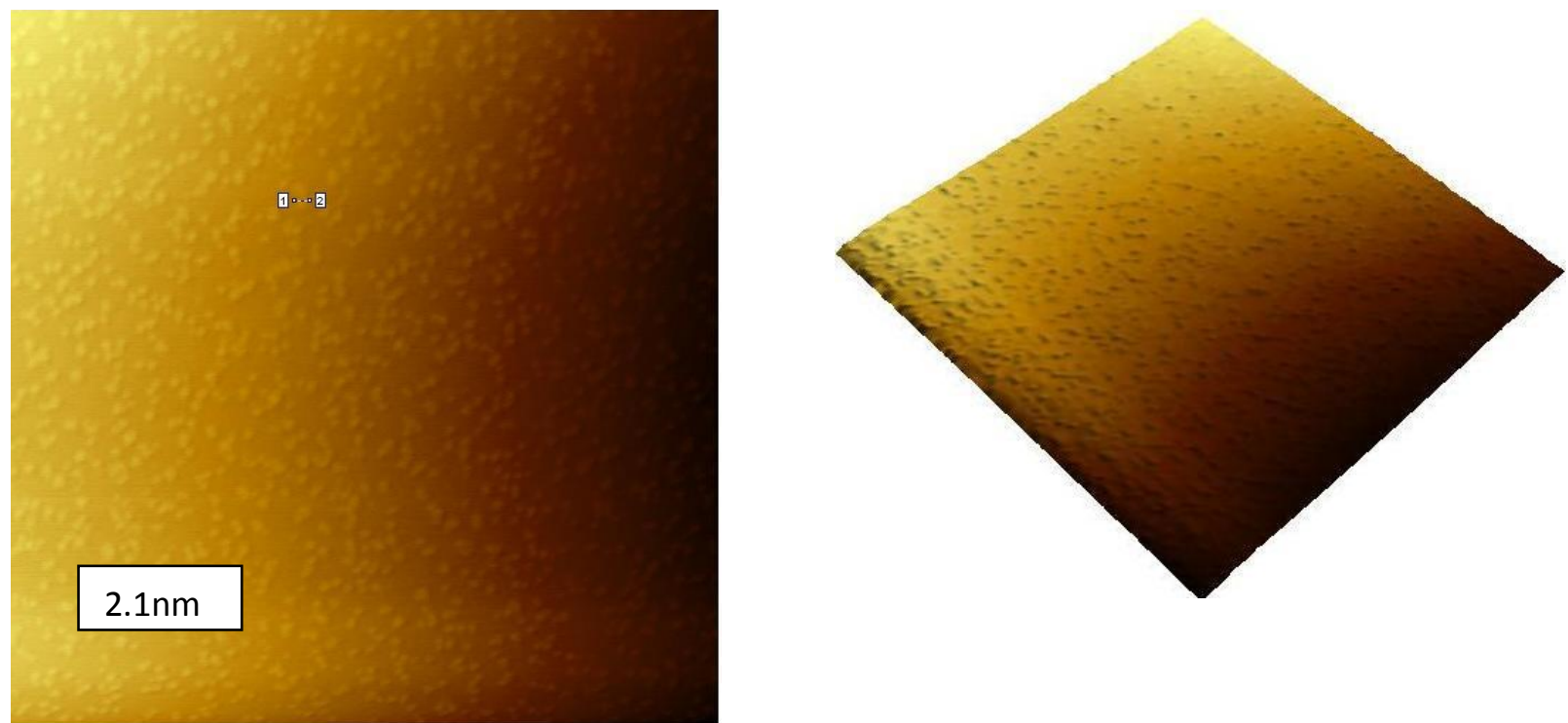

Fig 6 shows the AFM image of Pb-Cdse nanoparticles. It can .be concluded that the particles are spherical in shape and the size of the nanoparticle is $2.1 \mathrm{~nm}$.

XRD result of CdSe which is having peaks at 2 Theta angle of 26.52 and 42.465 and particle size is around $2.5 \mathrm{~nm}$ which are in agreement with report in the literature [5][6][7][8]. The crystal structure is hexagonal. The peak at 2 Theta angle of 50.154 is due to lead.

UV-VIS spectroscopy result of $\mathrm{Pb}-\mathrm{CdSe}$ nanopaticles which has absorbance at $684 \mathrm{~nm}$. The peak at $369.60 \mathrm{~nm}$ is due to change of spectrum from UV to VIS. The reason for not having a rise in peak is due to low concentration of sample used. The band gap energy corresponding to the absorbed wavelength can be calculated by $\mathrm{E}=\mathrm{hc} /$ wavelength, which comes out to be $2.9 \mathrm{eV}$.

From AFM result it can be concluded that particles are spherical in shape and the size is $2.1 \mathrm{~nm}$ which is agreement with the result from XRD. 


\section{CONCLUSION}

Single Electron Transistor are promising substitute for CMOS in charge sensing applications. SET made from Lead Cadmium Selenide have lower charging energy and hence can be operated at lower values of gate voltage. Moreover, Lead Cadmium Selenide is synthesized using a simple wet chemical method (polyol) and using minimum chemicals. Results from AFM and XRD are fruitful for the use of Lead Cadmium Selenide in SET. The Bandgap of Pb-CdSe found from the UV-VIS spectroscopy analysis is found out to be $2.9 \mathrm{eV}$. By proper designing of the tunneling junctions and by proper application of gate voltage we can control the charge on the island.

\section{ACKNOWLEDGEMENT}

The author would like to thank Nanotechnology Research Center, SRM University, Kattankulathur Campus for their continued support and help in characterizing the nanoparticles.

\section{REFERENCE}

[1] A. Boubaker, N. Sghaier, M. Troudi, a. Kalboussi, N. Baboux, and a. Souifi, "A new SIMPLORER model for single-electron transistors," Microelectronics Journal, vol. 38, no. 8-9, pp. 894-899, Aug. 2007.

[2] D. L. Klein, R. Roth, A. K. L. Lim, A. P. Alivisatos, and P. L. Mceuen, "A single-electron transistor made from a cadmium selenide nanocrystal," no. October, pp. 5-7, 1997.

[3] P. Hadley, G. Lientschnig, and M. Lai, "Single-Electron Transistors," pp. 1-8.

[4] P. B. Agarwal and a. Kumar, "Design and simulation of octal-to-binary encoder using capacitive single-electron transistors (C-SETs)," Microelectronics Journal, vol. 42, no. 1, pp. 96-100, Jan. 2011.

[5] M.Kavosh Tehrani, A.R Gholamiyankhak, N.Tavakkoli, H.Tavallali,"Synthesis and Characterization of CdSe Nanoparticles by Chemical Route."World Applied Science Journal (Supplement 1) 41-44, 2009

[6] J. Zhu, X. Liao, X. Zhao, and J. Wang, "Photochemical synthesis and characterization of CdSe nanoparticles," no. February, pp. 339343,2001 .

[7] K. Kandasamy, H. B. Singh, and S. K. Kulshreshtha, "Synthesis and characterization of CdS and CdSe nanoparticles prepared from novel intramolecularly stabilized single-source precursors," Journal of Chemical Sciences, vol. 121, no. 3, pp. 293-296, Jul. 2009.

[8] D. K. Dwivedi, V. Kumar, M. Dubey, and H. P. Pathak, "STRUCTURAL, ELECTRICAL AND OPTICAL INVESTIGATIONS OF CdSe NANOPARTICLES,” vol. 1, no. 6, pp. 21-25, 2011 